\title{
(K)ein Risiko für Mutter und Kind?
}

Fragestellung: Welche Aussagen können hinsichtlich der Messung medizinischer Parameter bei den Müttern und der Untersuchung kindlicher perinataler Besonderheiten bezogen auf die Einnahme von Antipsychotika in der Schwangerschaft getroffen werden?

Hintergrund: Schwangerschaften, in denen Antipsychotika eingenommen werden, haben zugenommen. Das mag daran liegen, dass mehr Frauen mit einer psychotischen Erkrankung schwanger werden und die Anwendung atypischer Neuroleptika bei depressiven und bipolaren Erkrankungen zugenommen hat. Es liegen noch ungenügende Informationen $\mathrm{zu}$ atypischen „neueren“ Antipsychotika in der Schwangerschaft vor. Es wird davon ausgegangen, dass das Fehlbildungsrisiko nicht erhöht ist. Genauere Beobachtungen zu medizinischen Komplikationen bei der Mutter und perinatal auftretende Probleme beim Neugeborenen wurden bisher nicht systematisch erhoben.

Patienten und Methodik: Die Studie wurde mit einem spezifischen epidemiologische Studiendesign für Kohortenstudien (high dimensional propensity score, HDPS) durchgeführt. Angewendet wird dieses empirische Verfahren bei einer großen Anzahl von Variablen, wie sie zum Beispiel bei Datenbanken im Gesundheitswesen vorliegen. Die Daten der vorliegenden Studie wurden aus Datenbanken des Gesundheitswesens der gesamten Provinz Ontario, Kanada, herangezogen. Ausgelesen wurden die Daten von Frauen, die ein Kind in einer Klinik zwischen 2003 und 2012 geboren

Vigod SN, Gomes T, Wilton AS et al. Antipsychotic drug use in pregnancy: high dimensional, propensity matched, population based cohort study. BMJ 2015; 350: h2298 hatten. Frauen, die an einer psychotischen Erkrankung litten, erhielten in etwa $70 \%$ Medikamente innerhalb eines speziellen Programms (Ontario Drug Benefit). Die personenbezogenen Daten wurden in der zugehörigen Datenbank erfasst und konnten für die Auswertung genutzt werden. Verschiedene weitere nationale Datenbanken wurden in die Berechnungen einbezogen. Ausgewertet wurden nur die Daten von Frauen, die mindestens zwei aufeinanderfolgende Verordnungen von Neuroleptika in der Schwangerschaft erhielten, wovon mindestens eine Verordnung in das erste oder zweite Trimester fallen musste. Die so ausgewählten Frauen $(n=1.021)$ wurden mit Frauen $(n=1.021)$ gematcht, die keine antipsychotische Medikation in der Schwangerschaft einnahmen. Berücksichtigt wurden alle Medikamentenverordnungen, erhobenen Diagnosen und Vorgänge $<365$ Tage vor Studieneinschluss. Endpunkte bezogen auf die Mutter waren Gestationsdiabetes, Gestationshypertonie, Präeklampsie, Eklampsie und venöse thrombembolische Ereignisse sowie bezogen auf das Kind Frühgeburten und abweichendes Geburtsgewicht. Weitere Endpunkte waren besondere mütterliche und neonatale Auffälligkeiten, Kontakte mit dem Gesundheitssystem und Sterblichkeit. Außerdem wurde zwischen der Verordnung von Quetiapin, Risperidon und Olanzapin unterschieden.

Ergebnisse: Die Verordnung von Quetiapin erfolgte bei 556 Frauen, von Olanzapin bei 166 und von Risperidon bei 112 Frauen. Diese zeigten kein signifikant höheres Risiko für Schwangerschaftsdiabetes, Gestationshypertension oder venöse thrombembolische Ereignisse. Die Rate der Frühgeburten und das durchschnittliche Geburtsgewicht unterschieden sich nicht statistisch relevant von Frauen ohne Antipsychotikaeinnahme. Auffallend war jedoch die hohe Assoziation mit operativen vaginalen Entbindungen und Geburtseinleitungen.

Schlussfolgerungen: Die Einnahme antipsychotischer Medikation in der Schwangerschaft hat wenig Einfluss auf mütterliche und kindliche Komplikationen. Jedoch sollte der Einsatz von Antipsychotika streng abgewogen werden, da im Vergleich zur Allgemeinbevölkerung häufiger Komplikationen auftraten.

\section{- Kommentar von Johanna Kunze, Chemnitz}

\section{Prospektive Daten fehlen ...}

Die mit einem HDPS-spezifischen Design durchgeführte Kohortenstudie erfüllt mit einem JADAD Score von 4 Punkten hohe methodische Ansprüche. Es kann gezeigt werden, dass es keinen signifikanten Einfluss von Neuroleptika in der Schwangerschaft auf die oben genannten mütterlichen und kindlichen Parameter gibt. Eine entscheidende Limitation der Studie stellt meines Erachtens der Einschluss von Frauen mit einem speziellen sozioökonomischen Status in den verwendeten Datenbanken dar und des Weiteren die nicht aufgeführte Verwendung weiterer psychotroper Substanzen zusätzlich zu der Verordnung von Neuroleptika. Interessant wäre aus meiner Sicht eine prospektive Beobachtung der Kinder über einen Zeitraum von mindestens drei Jahren, um eine verzö-

gerte Entwicklung nach Exposition mit psychotropen Substanzen auszuschließen.

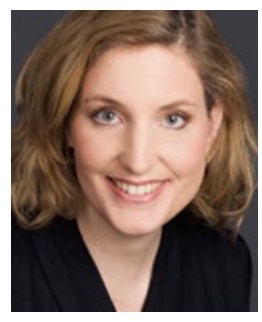

Dr. med. Johanna Kunze, Chemnitz

Fachärztin für Psychiatrie und

Psychotherapie, Sozialmedizin

Chefärztin Celenus Klinik Carolabad

E-Mail: j.kunze@carolabad.de 